Article

\title{
Effects of High-Order Simulations on the Simultaneous Stochastic Optimization of Mining Complexes
}

\author{
Joao Pedro de Carvalho *(D) and Roussos Dimitrakopoulos * \\ COSMO_Stochastic Mine Planning Laboratory, Department of Mining and Materials Engineering, \\ McGill University, Montreal, QC H3A 0E8, Canada \\ * Correspondence: joao.decarvalho@mail.mcgill.ca (J.P.d.C.); roussos.dimitrakopoulos@mcgill.ca (R.D.); \\ Tel.: +1-514-398-4755 (ext. 094748) (J.P.d.C.); +1-514-398-4986 (R.D.)
}

Received: 19 February 2019; Accepted: 26 March 2019; Published: 31 March 2019

\begin{abstract}
A mining complex is composed of mines, mineral processing streams, stockpiles, and waste facilities, which culminate with generated products that are delivered to customers. The supply uncertainty and variability of materials extracted from the mines, which flow through a mining complex to generate products, can be quantified through geostatistical simulations and can be used as inputs to the simultaneous optimization of mining complexes. A critical aspect to consider is that mineral deposits are characterized by spatially complex, non-Gaussian geological properties and multiple-point connectivity of high-grades, features that are not captured by conventional second-order simulation methods. This paper investigates the benefits of simultaneously optimizing a mining complex where the simulations of the mineral deposit are generated by a high-order, direct-block simulation approach. The optimized life-of-mine (LOM) production schedule is compared to a case in which the same setting is optimized by having the related simulations generated using a second-order simulation method. The comparison shows that the incorporation of simulations that reproduce the spatial connectivity of high-grades results in a more informed LOM production schedule. The sequence of extraction is driven by the spatial connectivity of high-grades, resulting in a mill throughput with better material quality and reduced waste extraction. Furthermore, the discounted cash-flow increases by more than $5 \%$ as compared to the case in which the second-order simulations are used.
\end{abstract}

Keywords: simultaneous optimization of mine complexes; high-order simulation; direct block support simulation; connectivity of high-grades

\section{Introduction}

A mining complex can be perceived as a transfer function that entails complex interactions, starting with the extraction of materials from the mines through to their transformation into saleable products, passing through different processes in the mineral value chain [1,2]. It can include, for example, multiple mines, various elements and material types, stockpiles, tailings facilities, waste dumps, processing plants and transportation systems [3]. The simultaneous stochastic optimization of a mining complex maximizes the global value of the mining operation by integrating all of the components in a single framework, while also including the uncertainty and variability of materials sourced from the mines as a set of stochastically generated realizations [1,2,4-7].

The modeling of large mining operations as an integrated mathematical model has advanced over the last three decades. Newmont Mining Corporation pioneered the area, proposing a linear programming approach to the massive gold mining complex in Nevada [8]. BHP also contributed 
significant improvements with the development of the Blasor optimizer, BHP's in-house software that was applied to the Yandi mine complex in Australia [9], optimizing the life-of-mine extraction sequence from multiple pits. Additional applications have extended the software to optimize and assess more complex requirements [10,11]. Whittle [12] and Whittle [13] describe an industry standard tool to sequence the extraction of material from multiple deposits in operations with complex blending and processing requirements. Topal and Ramazan [14] present an approach based on linear network flow to optimize a mining complex in Western Australia with more than 100 pits and 13 processing facilities. Khan and Asad [15] propose a mixed integer linear programming model extending the standard cut-off grade strategy used in the industry for the case of multiple processing streams. Although the methods mentioned above represent significant efforts to integrate the multiple components of a mining complex in order to capitalize on synergies, simplifications exist. Conventional approaches optimize the mining complex by solving each step or component sequentially; thus, they do not benefit from the interaction and cooperation of different components, which leads to suboptimal solutions for a value chain as a whole $[1,3]$. Another limitation in the studies to date is the aggregation of mining blocks into larger decision variable units, such as panels, which is done to make the optimization simpler but can misrepresent the mining selectivity. Moreover, the uncertainty in material supply coming from the mines has been long recognized as the primary cause of technical risk in mining operations [16] and, if left unmanaged, leads to unexpected deviations in production targets [17-20].

The spatial uncertainty and variability of attributes in geosciences can be quantified via geostatistical simulations [21-26], which are founded on the concept of random fields. The sequential simulation approach is an alternative to assess these attributes at each unsampled location of a three-dimensional orebody model through Monte Carlo sampling from a conditional distribution function [21-23]. Traditional simulation methods are based on the second-order statistics, namely, mean and covariance (variogram), where sequential Gaussian simulation (SGS) $[23,27,28]$, sequential indicator simulation (SIS) [23] and sequential direct block simulation [29,30] are some examples. However, geological attributes of spatially distributed phenomena are represented by complex non-Gaussian and non-linear spatial connectivity of low- and high-grades. Using only up to second-order statistical statistics in spatial simulations is not sufficient to fully describe complex geological attributes [31]. In addition, Gaussian simulation methods maximize spatial disorder (maximum entropy) beyond the imposed variogram in the realizations generated [32], thus preventing a more realistic quantification of the connectivity of high-grades.

Multiple-point statistics (MPS) simulation methods have been introduced in an attempt to address the above limitations [33-42]. MPS methods replace the random field model with a geological analogue or training image (TI) and facilitate the reproduction of complex curvilinear and other geologic features in the realizations generated, while avoiding distributional assumptions. A consequence of extracting patterns from a TI is that generated realizations are TI-driven and their spatial statistics may be different from those of the available data, particularly when relatively dense drillhole data sets are available $[43,44]$. As a natural extension of second-order methods, the high-order simulation framework [45-50] can reproduce very complex non-linear geometries and spatial statistics from data by explicitly calculating high-order spatial cumulants. The generated realizations present a more realistic and structured connectivity of high-grades (lower entropy) compared to traditional methods, as shown in the example in Figure 1.

The appropriate characterization of spatial connectivity and its impact in flow modelling are well-studied subjects in the context of reservoirs and aquifers [32,51-53]. In the mining context, some studies have shown that the use of different simulation frameworks impacts the output of transfer functions [54,55]. Geostatistical simulations have been effectively incorporated into state-of-the-art simultaneous stochastic optimization of mining complex frameworks [1,2,4-6,56,57]. The next step is to investigate the effects of high-order simulation models in this optimization framework. 


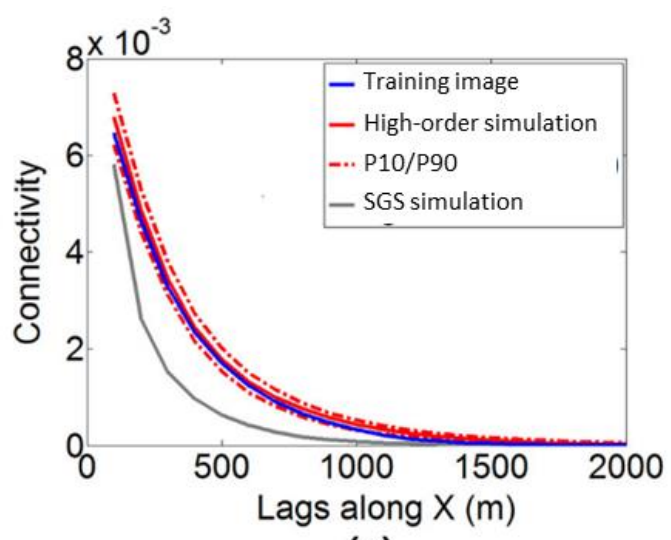

(a)

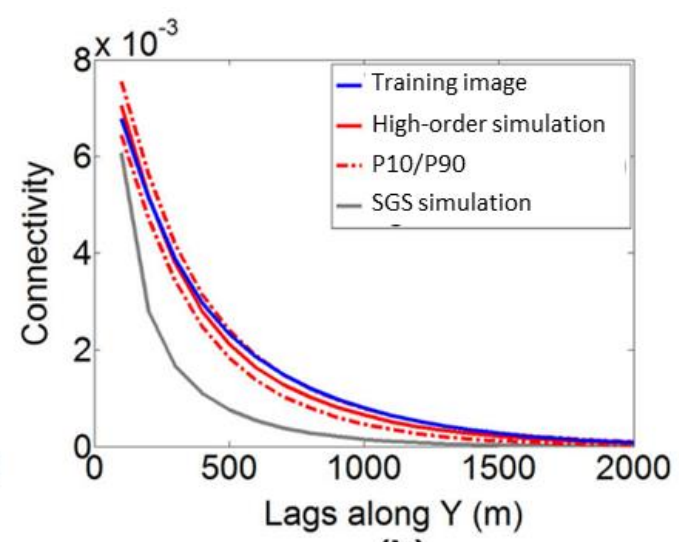

(b)

Figure 1. Connectivity of high-grades along $X(\mathbf{a})$ and $Y(\mathbf{b})$ direction, calculated for the 99th percentile from the data distribution. Figure adapted from Minniakhmetov et al. [49]. P10 and P90 represent the 10th and 90th percentiles of the reported values, respectively.

This paper presents an application of the high-order direct block-support simulation method [48] used as an input to the simultaneous stochastic optimization of a simplified mine complex, using the framework proposed by Goodfellow and Dimitrakopoulos [6]. As a means of comparison, the same mine complex is optimized using simulations obtained with a second-order method. The differences in life-of-mine production schedules and related production forecasts, optimized for each case, are analyzed and discussed. The following sections provide a brief description of the high-order direct block simulation framework and the optimization model. This is followed by a comprehensive analysis of the different outcomes from the two approaches, and finally, a conclusion section is presented.

\section{Materials and Methods}

\subsection{Modeling a Mineral Deposit Using Geostatistical Simulations}

Considering a random function $(\mathrm{RF}) Z^{P}\left(x_{i}\right), x_{i} \in R^{d}$, where $x_{i}$ represents the location of the point support grid to be simulated in the domain $D \in R^{d}$ and $z_{i}^{P}$ a realization of $Z^{P}\left(x_{i}\right)$. The set of initial data is given by $d_{n}=\left\{z^{p}\left(x_{k}\right), k=1, \ldots, n\right\}$ and represents the values obtained from the exploration data. Now consider the set $\Lambda_{i}$ with the data and previously simulated nodes, i.e., $\Lambda_{0}=\left\{d_{n}\right\}$ and $\Lambda_{i}=\left\{\Lambda_{i-1} \cup Z\left(x_{i}\right)\right\}$. Thus, under the sequential simulation framework $[23,27,52,58,59]$ the global conditional distribution can be decomposed in the multiplication of the univariate distributions

$$
f\left(x_{1}, \ldots, x_{n} ; z_{1}^{P}, \ldots, z_{n}^{P} \mid \Lambda_{0}\right)=f\left(x_{1} ; z_{1}^{P} \mid \Lambda_{0}\right) * \ldots * f\left(x_{n} ; z_{n}^{P} \mid \Lambda_{n-1}\right)
$$

\subsubsection{High-Order Direct Block-Support Simulation}

Instead of simulating the entire deposit at the point support discretization, the work by de Carvalho et al. [48] presents an alternative method that allows the generation of high-order simulations directly at the block scale. Assume $z_{i}^{v}$ is a realization of the RF $Z^{v}\left(v_{i}\right)$ at the block support scale, defined in the same domain $D \in R^{d}$, where $v_{i}$ represents the centroid of the block in consideration. Additionally, the conditional distribution, $f\left(v_{i} ; z_{i}^{v} \mid \Lambda_{0}, \Lambda_{i-1}^{v}\right)$, is derived directly at the block support scale, where $\Lambda_{j}^{v}=\left\{z^{v}\left(v_{m}\right), m=1, \ldots, j\right\}$ is the set of previously simulated blocks.

The cross-support $f\left(v_{i} ; z_{i}^{v} \mid \Lambda_{0}, \Lambda_{i-1}^{v}\right)$ distribution is simplified according to Bayes rule as

$$
f\left(v_{i} ; z_{i}^{v} \mid \Lambda_{0}, \Lambda_{i-1}^{v}\right)=\frac{f\left(v_{i}, \lambda_{0}, \lambda_{i-1}^{v} ; z_{i}^{v}, \Lambda_{0}, \Lambda_{i-1}^{v}\right)}{\int f\left(v_{i}, \lambda_{0}, \lambda_{i-1}^{v} ; z_{i}^{v}, \Lambda_{0}, \Lambda_{i-1}^{v}\right) d v_{i}}
$$


where $\lambda_{0}$ and $\lambda_{i-1}^{v}$ are the set of locations of $\Lambda_{0}$ and $\Lambda_{i-1}^{v}$, respectively. For simplicity, $f\left(v_{i}, \lambda_{0}, \lambda_{i-1}^{b} ; z_{i}^{v}, \Lambda_{0}, \Lambda_{i-1}^{b}\right)$ is referred to herein as

$$
\begin{aligned}
f\left(v_{i}, \lambda_{0}, \lambda_{i-1}^{v} ; z_{i}^{v}, \Lambda_{0}, \Lambda_{i-1}^{v}\right) & =f(z_{i}^{v}, \underbrace{\left\{z_{1}^{p}, \ldots, z_{n}^{p}\right\}}_{\text {exploration data }}, \underbrace{\left\{z_{1}^{v}, \ldots, z_{i-1}^{v}\right\}}_{\text {previously simulated blocks }}) \\
& =f\left(z_{1}^{v}, \ldots, z_{i}^{v}, z_{1}^{p}, \ldots, z_{n}^{p}\right)
\end{aligned}
$$

Thus, the above is approximated as

$$
\begin{gathered}
f\left(z_{1}^{v}, \ldots, z_{i}^{v}, z_{1}^{p}, \ldots, z_{n}^{p}\right) \approx \\
\sum_{k_{1}^{v}}^{\omega} \ldots \sum_{k_{i}^{v}}^{\omega} \sum_{1}^{\omega} \ldots \sum_{k_{n}^{p}}^{\omega}\left[L_{k_{1}^{v}} \ldots k_{i}^{v} k_{1}^{p} \ldots k_{n}^{p} \varphi_{k_{1}^{v}}\left(z_{1}^{v}\right) \ldots \varphi_{k_{i}^{v}}\left(z_{i}^{v}\right) \varphi_{k_{1}^{p}}\left(z_{1}^{p}\right) \ldots \varphi_{k_{n}^{p}}\left(z_{n}^{p}\right)\right]
\end{gathered}
$$

where $\varphi_{m}(\cdot)$ belongs to the set of Legendre-like orthogonal splines [49,60], and the coefficient $L_{i \ldots . . j k \ldots l}$ is approximated experimentally by

$$
L_{i \ldots j k \ldots l} \approx \mathbb{E}\left[\varphi_{i}\left(z_{1}^{v}\right) \ldots \varphi_{j}\left(z_{n_{v}}^{v}\right) \varphi_{k}\left(z_{1}^{p}\right) \ldots \varphi_{l}\left(z_{n_{p}}^{p}\right)\right]
$$

The method utilizes a TI represented in both support sizes, point and block. Thus, having a spatial template obtained with the block to be simulated and neighboring values, both at the block and point support, this TI is scanned searching for replicates of the template in consideration. The algorithm for block support high-order simulation can be summarized as:

1. Upscale the TI inputted at point support to block support.

2. According to the sequential simulation framework, define a random path to visit all the unsampled block locations.

3. At each block location:

a. Find the closest point and block support values for conditioning.

b. Obtain a spatial template configuration formed by the block to be simulated and related conditioning values.

c. Scan the TI searching for replicates of the above template.

d. Calculate the spatial cross-support coefficients $L_{i \ldots . . j k \ldots l}$ using Equation (5).

e. $\quad$ Derive the conditional cross-support joint probability density function $f\left(v_{i} ; z_{i}^{v} \mid \Lambda_{0}, \Lambda_{i-1}^{v}\right)$ by first calculating the joint distribution in Equation (4), then normalizing the distribution in Equation (2).

f. Draw a uniform value from $[0,1]$ to sample $z_{i}^{v}$ from the conditional cumulative distribution derived from the above.

g. Add $z_{i}^{v}$ to the simulation grid at block support at location $v_{i}$ to be used as conditioning value to the simulation of a subsequent block.

4. Repeat steps 2 and 3 to generate additional realizations.

\subsubsection{Sequential Gaussian Simulation}

The case study in Section 3 also applies the sequential Gaussian simulation (SGS) $[23,27,61]$. The method assumes that the conditional distribution $f\left(x_{i} ; z_{i}^{p} \mid \Lambda_{i-1}\right)$ is Gaussian, which facilitates the simulation process, since its approximation only requires the definition of two parameters, namely, mean and variance. For this reason, the original data is transformed into the Gaussian space typically by a graphical transformation; and at every node, the method solves a kriging system to obtain the 
posterior mean and variance. The simulation is performed at the point support scale with a subsequent re-blocking procedure to generate block-support models.

\subsection{Mathematical Formulation of the Simultaneous Optimization of Mining Complexes}

The current study uses the simultaneous optimization of mining complexes model proposed in $[1,6]$, which is summarized as follows. In this setting, the mine is discretized into mining blocks indexed in $b \in B$. The framework considers that each block has simulated attributes, such as grades and material types, which will denote a stochastic scenario $s \in S$. The extraction of each block $b$ in period $t \in T$ incurs a mining cost $M C_{b, t}$, but its extraction can only happen if the set of predecessors $O(b)$ has already been extracted. Once extracted, the material can flow from the mine to a stockpile or a destination, such as waste pile, leach pad or mill (processors $i \in P$ ). The cost associated with material transportation is given by $T C_{i, a, t}$. The amount of property $a$ in location $i$, period $t$ and scenario $s$ is quantified in $v_{a, i t, t s}$. Material that flows from the mine to a location, for example, ore tonnage, is indexed in $p \in \mathbb{P}$, whereas transformations, potentially non-linear ones such as recovery, are indexed in $h \in \mathbb{H}$. Thus, $p_{h, t, s}$ denotes the unitary value of selling the material property $h$, in period $t$ and scenario s. $P C_{i, p, t}$ is the processing cost of treating material property $p$, in location $i$ at the period $t$. The set of production targets is represented by $\mathbb{P}_{c}$, where deviations are quantified in $d_{i, a, t, s}^{ \pm}$and penalized by the $\operatorname{cost} c_{i, a, t}^{ \pm}$. The model also incorporates smoothing and sink constraints $[62,63]$, where deviations of these targets are quantified in and penalized by $d_{b, t}^{\text {smooth }}, d_{b, t, v}^{\text {sink }}$ and $c_{b, t}^{\text {smooth }}, c_{b, t}^{\text {sink }}$, respectively. Additional sets of constraints such as slope, reserve, capacities, destination policy and processing stream constraints are detailed in [6].

There are three types of decision variables in the described model. Extraction sequence $\left(x_{b, t} \in\{0,1\}\right)$ returns 1 if the block $b$ is extracted at the period $t, 0$ otherwise. Destination decisions $\left(z_{g, j, t} \in\{0,1\}\right)$ define where to send a group of material $g$ to the destination $j$ at the period $t$. These groups are defined in a similar manner to what is described in [11], where pre-defined grade bins are inputted, and the optimizer decides the single element optimal cut-off grade boundaries. Processing stream decisions $\left(y_{i, j, t, s} \in[0,1]\right)$ define the proportion of material that flows from location $i$ to destination $j$, in period $t$ and scenario $s$.

The objective function in Equation (6) maximizes the value of selling the products in the mineral value chain, while minimizing deviation from production targets.

$$
\begin{gathered}
\max \frac{1}{\|\mathbb{S}\|}\left\{\sum_{s \in S} \sum_{t \in T}\{\underbrace{\sum_{i \in P}^{\sum_{h \in \mathbb{H}} p_{h, t, s} * v_{h, i, t, s}}-\underbrace{\sum_{i \in P} \sum_{p \in \mathbb{P}}\left(P C_{i, p, t}+T C_{i, p, t}\right) * v_{p, i, t}}_{\text {Part II }}}_{\text {Part I }}\right. \\
-\underbrace{\sum_{i \in P} \sum_{p \in \mathbb{P}_{c}}\left(c_{i, p, t}^{+} * d_{i, p, t, s}^{+}+c_{i, p, t}^{-} * d_{i, p, t, s}^{-}\right)}_{\text {Part III }}\}\}-\underbrace{\sum_{t \in T} \sum_{b \in B}\left(M C_{b, t} * x_{b, t}+c_{b, t}^{\text {smooth }} * d_{b, t}^{\text {smooth }}\right)}_{\text {Part IV }} \\
\underbrace{\sum_{t \in T} \sum_{b \in B} \sum_{v \in V_{b}}\left(c_{b, t}^{\text {sink }} * d_{b, t, v}^{\text {sink }}\right)}_{\text {Part } V}
\end{gathered}
$$

Part I of Equation (6) considers the discounted cash flow obtained by selling the products in the value chain. Part II minimizes the processing cost at each processing facility and the transportation costs involved. The third Part is related to the cost of deviating from processing and mining capacities, respectively. Part IV minimizes the deviation from schedule smoothness and mining 
costs, while Part V aims to reduce deviations from sink rate constraints, respectively. Note that geological risk discounting [62] is applied to all penalty costs associated with production target, i.e.,

$$
c_{i, a, t}^{ \pm}=\frac{c_{i, a}^{ \pm}}{(1+r)^{t}}
$$

where $r$ is the geological discount rate.

The simultaneous optimization of a mining complex framework presented above is very general and allows for the integration of different sources of uncertainty; in addition, it addresses the non-linearity related to materials blending and stockpiling, as well as transformations that are due to refining materials to output products. The above optimization approach leads to instances with multi-million binary decision variables that cannot be solved with commercial solvers, such as CPLEX. Metaheuristic algorithms offer a practical alternative and have proven to be an efficient solving approach for the stochastic optimization of mines and mining complexes $[6,56,64-66]$. The solution approach used in the current paper is from Goodfellow and Dimitrakopoulos [1,6].

\section{Results and Discussion}

The first part of the case study relates to the simulation of the grades of a gold mine. The deposit covers an area of approximately $4.5 \mathrm{~km}^{2}$ and extends to a depth of $400 \mathrm{~m}$. The three-dimensional orebody model is composed of 510,800,10 $\times 10 \times 10 \mathrm{~m}^{3}$ mining blocks. The available data come from 2344 drillholes that are spaced at about $35 \mathrm{~m}$ apart and include 40,762, $10 \mathrm{~m}$ long gold composites. A set of 15 high-order simulations are generated directly at the scale of mining blocks using a training image generated from blasthole data obtained at a $5 \mathrm{~m}$ spacing. Each high-order simulation required approximately $6 \mathrm{~h}$ on an Intel ${ }^{\circledR}$ Core $^{\mathrm{TM}}$ i7-7700 CPU with $3.60 \mathrm{GHz}$ and 16GB of RAM, running on Windows 7. For comparison, a set of simulations based on the second-order statistics is also generated using traditional sequential Gaussian simulation (SGS) $[23,27,61]$. Realizations generated at the point-support scale are rescaled to block support using a discretization of 25 nodes per block.

\subsection{Results, Comparisons and Effects of High-Order and Second-Order Simulations}

First, to provide a common ground for comparison, Figure 2 shows the grade-tonnage curve for both simulation frameworks with equivalent overall reporting and quantification of related uncertainty. The graph shows very similar proportions regarding tonnages and grades over the deposit. Although the metal quantity is very comparable in both cases, how each method connects these elements in space can be very different, especially at the high-grade values, as noted earlier in Figure 1. Figure 3 displays cross sections of both second-order and high-order realizations of the deposit, where the high-grade zones are highlighted with red circles. It is possible to visualize the effect of the maximum entropy property over the second-order simulations, which is enhanced by the fact that the simulation process was performed in the Gaussian space. The grades displayed by the simulations generated with SGS are visually more dispersed than those generated using high-order simulations. This connectivity can be quantified [52] and it is presented in Figure 4. Please note that, in all figures, P10 and P90 represent the 10th and 90th percentiles of the reported values, respectively. For the connectivity plot, the cut-off applied is $5 \mathrm{~g} /$ ton, corresponding to the 99th percentile of the grade distribution, as the focus of the comparison is on the high-grades. In the NE direction, the second-order realizations are consistently less connected than the high-order realizations for all lags. The difference becomes more pronounced in the NE direction and at the $45^{\circ}$ dip, with a considerable gap evident between both simulation methods. As the high-grade mineralization drives the mine production schedule, this plays a vital role in the optimization of the mining complex. 


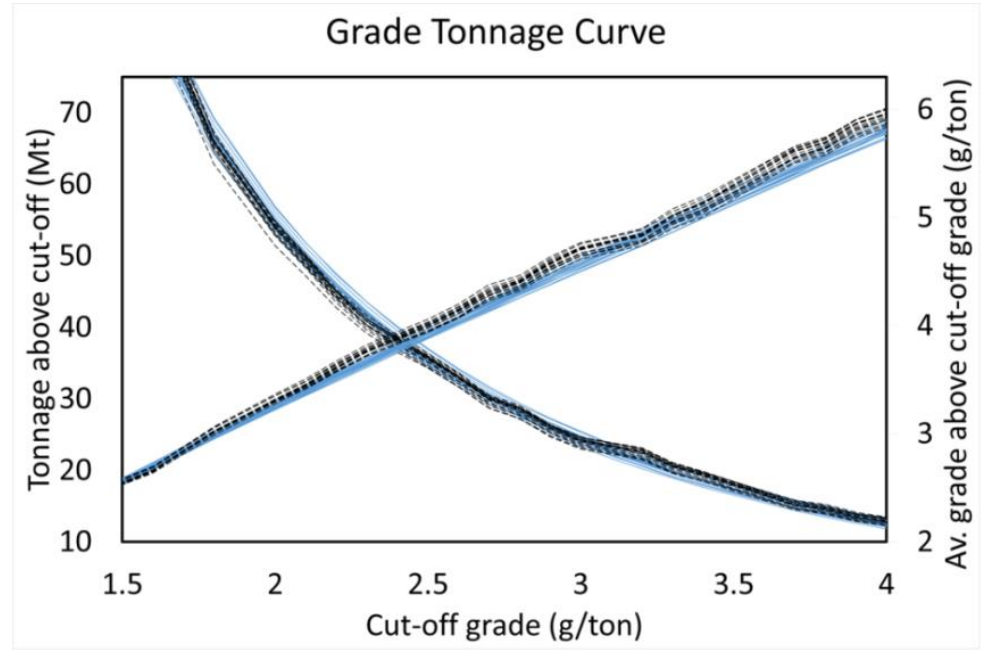

\section{Cloud of simulations based on high-order - - second-order}

Figure 2. The grade-tonnage curve of the gold deposit for SGS (second-order) and high-order simulations.

a) High-order simulations
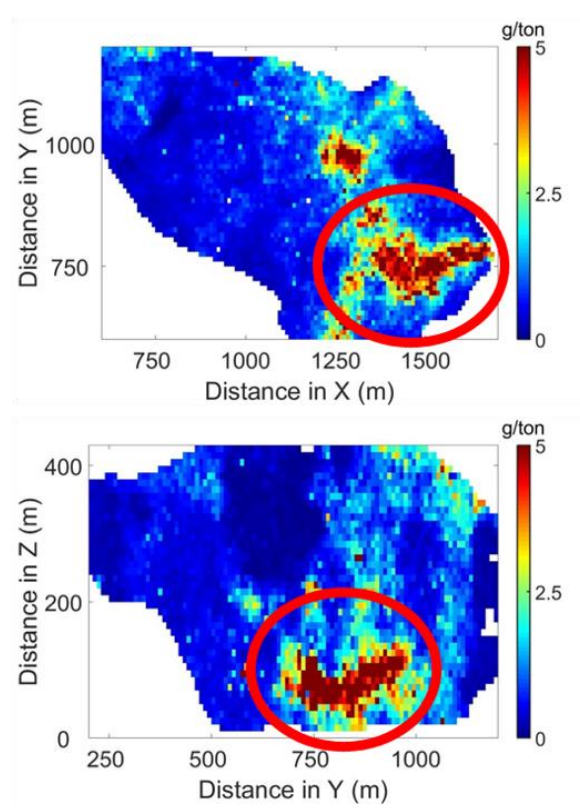

b) second-order simulations
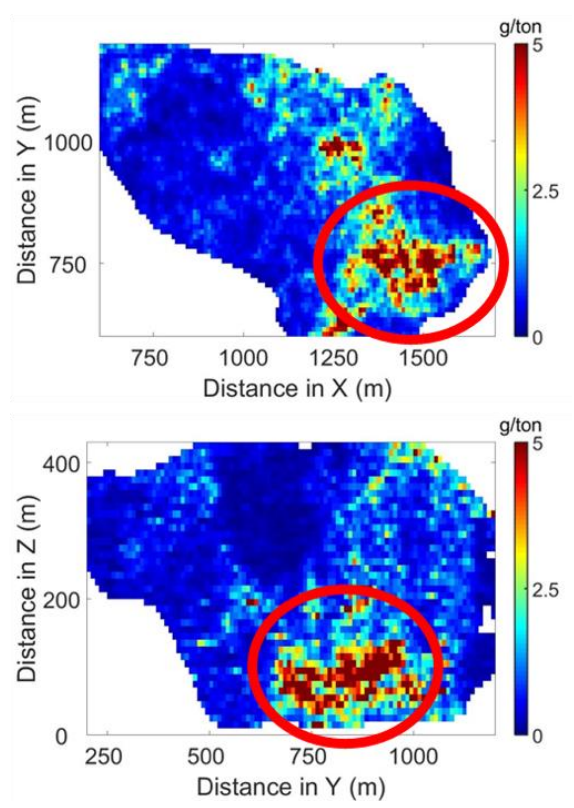

Figure 3. Cross-sections of the simulations, obtained from high-order (a) and second-order (b) based methods, highlighting differences in connectivities of high-grades. The red circles highlight the high-grade zones. 
a)

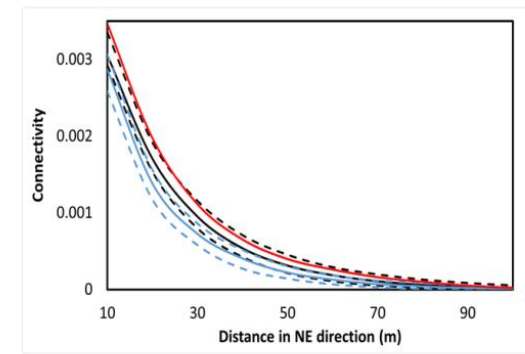

P10/P90 =--: $\quad$ High-order simulations - b)

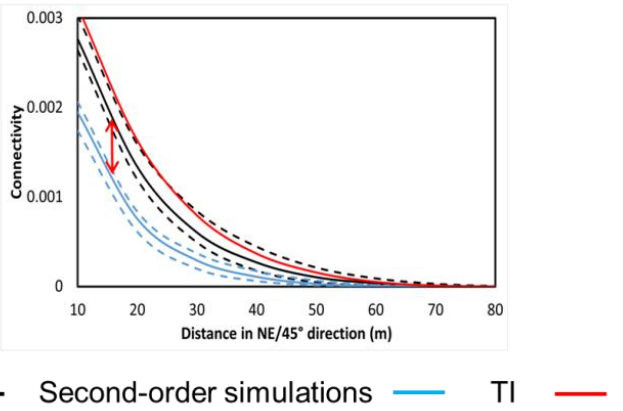

Figure 4. Connectivity of simulated realizations at (a) NE direction; (b) NE/45 direction. The arrow highlights the gap between the outcomes from the two methods.

\subsection{LOM Production Schedule Optimization and Forecasting}

The mining complex considered in the test case consists of a single gold mine, where ore and waste material can flow to the following destinations: Leach pad, ore stockpile, waste dump, and a mill circuit (Figure 5). Both the mill and the leach pad process ore to generate sellable products, while encountering different costs and recoveries. Since the high-grade materials are typically processed at the mill, having a substantially higher impact on cash flows, this processing stream receives more attention in this paper. Doing so simultaneously allows for investigating the impact of the high-grade spatial connectivity generated by the two simulation methods examined. The critical parameters for the optimization, displayed in Table 1, are kept the same in both cases. The uncertainty in the material properties sourced from the mine is quantified by the set of simulations generated and is used as input for the optimization framework. The mining complex is first optimized using simulations generated by the high-order direct block support method, which is referred to throughout the remainder of the paper as Case 1. The same mining complex setting is then optimized, using the set of simulations generated by the second-order Gaussian simulation method. The result of this optimization is referred to as Case 2.

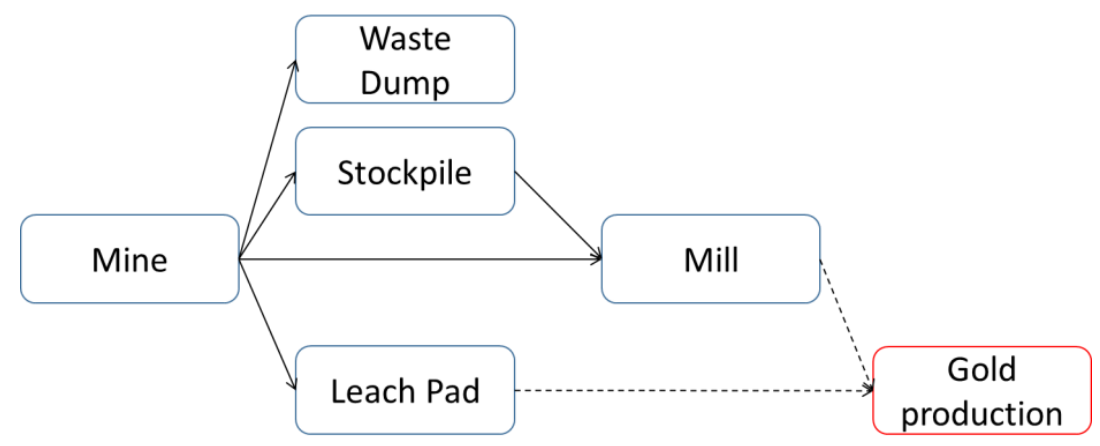

Figure 5. Flow diagram of the mine complex configuration.

The life of mine production schedules for Case 1 and 2 are optimized and results are discussed below. Cross sections of the LOM production schedules optimized for each case are displayed in Figures 6 and 7 along West-East and North-South directions, respectively. The areas with the same color represent the same period of extraction, and they highlight that the sequences of extraction obtained differ considerably, which is not surprising given the differences in the two simulation methods used. Cross sections of Case 1 show that the sequence of extraction follows a clear direction, highlighted by the red arrow. Note that this trend in the extraction sequence is amplified in the direction where the difference in connectivity is more evident, recall from Figure 4 . The higher continuity of high-grades drives the schedule towards areas with more connected ore materials so that they can be processed together. 
Table 1. Main parameter used in the optimization.

\begin{tabular}{cc}
\hline Parameter & Specification \\
\hline Mine capacity & $30 \mathrm{Mtpa}$ \\
Mill capacity & $8.25 \mathrm{Mtpa}$ \\
Leach pad capacity & $10 \mathrm{Mtpa}$ \\
Sink rate & $60 \mathrm{~m} / \mathrm{year}$ \\
Mining radius & $60 \mathrm{~m}$ \\
Mining cost & $1.6 \$ / \mathrm{ton}$ \\
Milling cost & $7.84 \$ / \mathrm{ton}$ \\
Leaching cost & $2.30 \$ / \mathrm{ton}$ \\
Refining cost & $12.97 \$ / \mathrm{oz}$ \\
Gold price & $1250 \$ / \mathrm{oz}$ \\
Discount rate & $10 \%$ \\
Geologic risk discounting & $10 \%$ \\
\hline
\end{tabular}

a) Case 1 (high-order simulations)

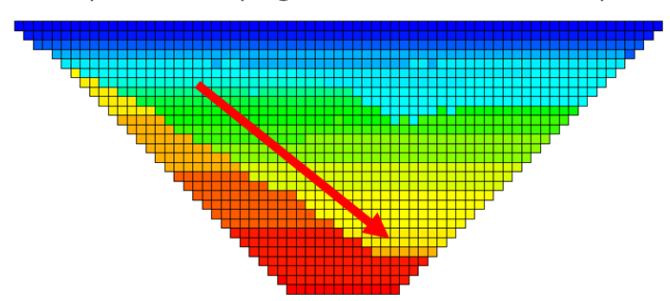

b) Case 2 (second-order simulations)

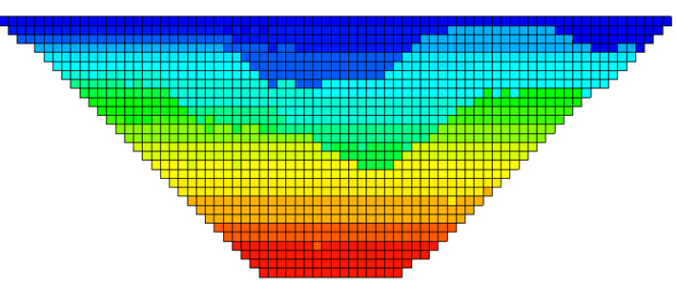

\section{Period (years) $1 \quad 5 \quad 10 \quad 15 \quad 20$}

Figure 6. Cross-section of the life-of-mine (LOM) production schedule (plane East-West) obtained in (a) Case 1 and (b) Case 2. The red arrow shows the preferential mining direction along the direction of higher continuity of high-grades.

a) Case 1 (high-order simulations)

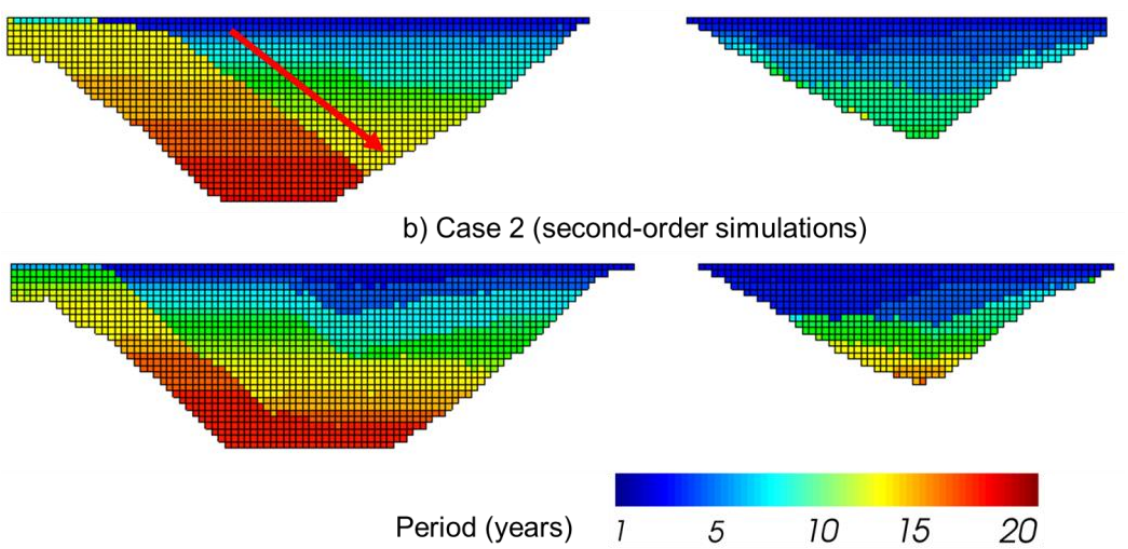

Figure 7. Cross-section of the LOM production schedule (plane North-South) obtained in (a) Case 1 and (b) Case 2. The red arrows show the preferential mining direction in the direction of higher continuity of high-grades.

Figure 8 shows horizontal sections of the LOM production schedules generated and the differences in the sequences of extraction are again evident. Additionally, these sections show variations in the extension of the ultimate pit limits (UPL). The red circles highlight how much larger the UPL is in Case 2. As second-order simulations methods represent high-grade material as being more scattered, it is logical that the pits have to be larger to encompass all of the ore to be processed, resulting in a higher waste extraction, as shown in Figure 9a. These differences are of particular interest, since after 
the optimization is complete, infrastructure, such as ramps, access points, equipment placement and facility locations, reduces the flexibility to change the schedule. This optimization opportunity can only be achieved if the degree of connectivity of high-grades is correctly modeled.

a) Case 1 (high-order simulations)

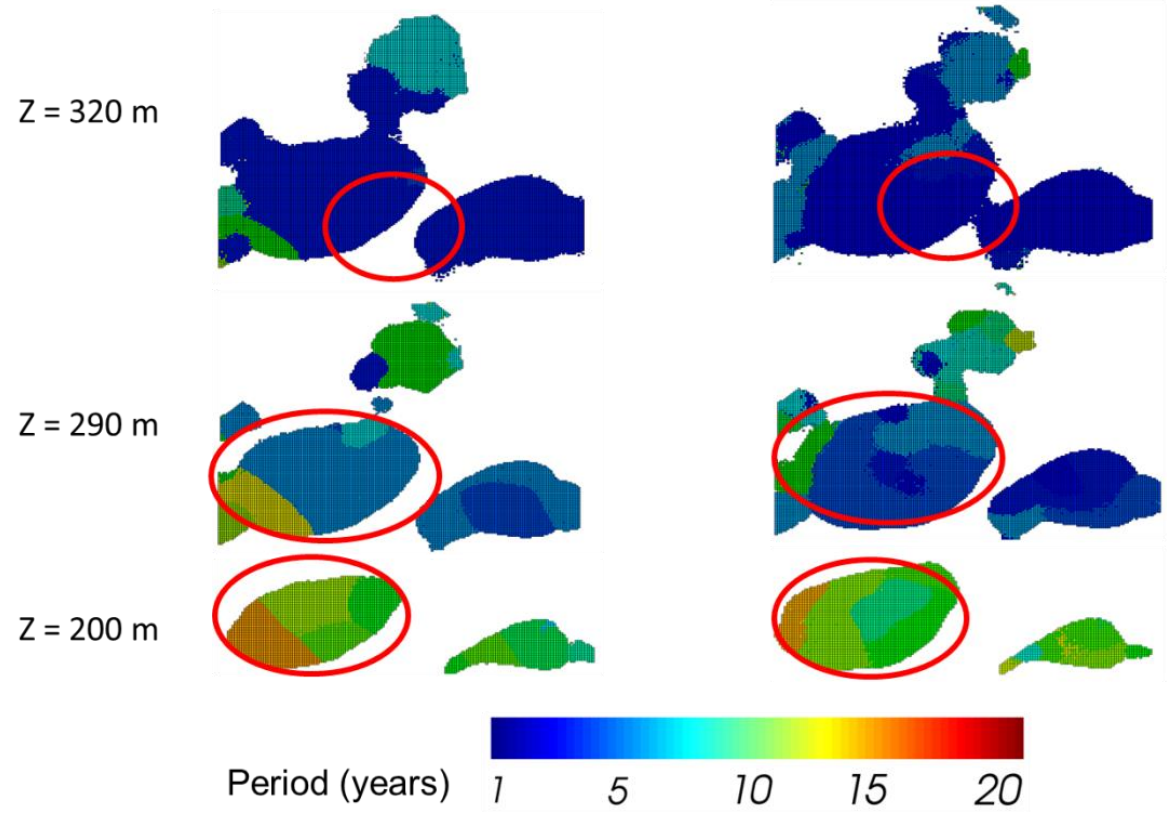

Figure 8. Horizontal sections of the mine production schedule at different elevations for (a) Case 1 and (b) Case 2. The red circles highlight the differences in pit sizes.

a)

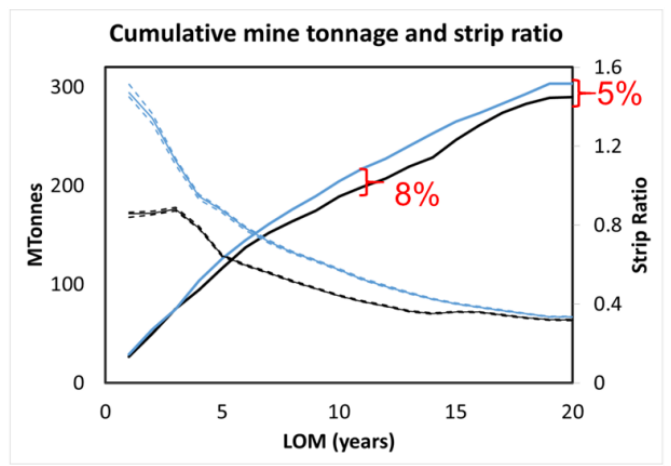

P10/P90 =-- Case $1-$ b)

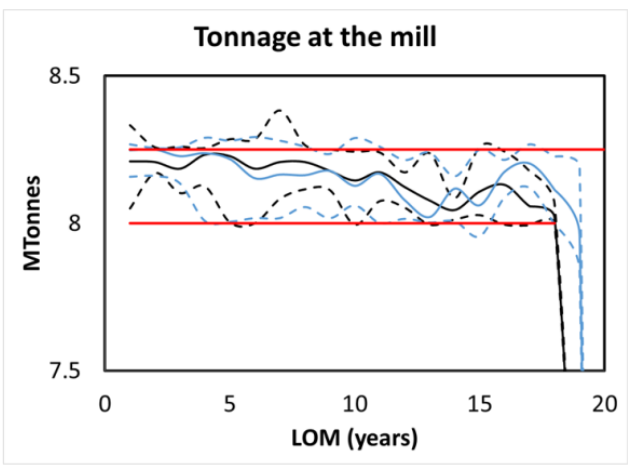

Case $2-\quad$ UB/LB Target -

Figure 9. Production target assessments: (a) Cumulative mine tonnage and strip ratio; (b) mill throughput with upper and lower bounds. P10 and P90 representing the 10th and 90th percentiles, respectively.

Once the production schedules are generated, the risk assessment of achieving production forecasts is calculated by pushing another set of simulations through each LOM schedule and by evaluating related forecasts. The results are presented in terms of P10 and P90, representing the 10th and 90th percentiles of related performance indicators, respectively. Regarding production targets and forecasts, Figure 9a shows the total tonnage mined over the LOM and the cumulative strip ratio for both cases. The production schedule obtained in Case 2 represents, in total, $5 \%$ more material mined than in Case 1. This difference reaches $8 \%$ at the end of the 10th year to ensure a similar throughput at the mill, Figure $9 \mathrm{~b}$. Mining more, in this case, translates to higher waste production, which is quantified by the higher strip ratios presented by Case 2 . This can be explained by the spatial disorder 
(maximum entropy) that Gaussian-based approaches generate with respect to high-grades. Having ore blocks less connected in space forces the optimizer to mine more dispersed high-grade values (mining blocks), so as to provide a consistent feed rate to the mill; this also leads to different total tonnages of materials mined and the differences in UPL shapes, shown in Figure 8. On the other hand, if the optimizer receives more realistic information regarding spatial grade connectivity, the LOM production schedule, such as in Case 1, pursues high-grades more efficiently.

Although the mill's throughput is kept reasonably constant throughout the LOM in both instances, the dissimilarities regarding metal content are stressed in Figure 10. Case 1 can feed the mill with a higher head grade for the majority of the LOM, as shown in Figure 10a. As the optimizer encounters better-connected zones of high-grade, it can bring their extraction to the same period so they can be processed together, increasing the average feed grade at the mill and recovering more ounces earlier, as shown in Figure 10b. Case 1 shows an ounces profile that is consistently higher for the first 17 years; this difference reaches $7 \%$ after the 10th year. Case 2 produces, after the 20th year, $2 \%$ more gold, but this is not significant due to the effect of discounting and the time value of money. Recovering more ounces sooner brings more cash flow earlier to the operation, which positively impacts the net present value (NPV). Summing up the joint effects of correctly meeting production targets, mining less waste and producing more gold earlier results in a considerable increase in NPV, as shown in Figure 10c. By producing more metal and less waste, the LOM production schedule obtained in Case 1 generates in a total of $5 \%$ higher NPV than Case 2, and 16\% higher in the initial ten years. The difference is substantial and improves financial returns at the early stages of the development of the mine.

a)

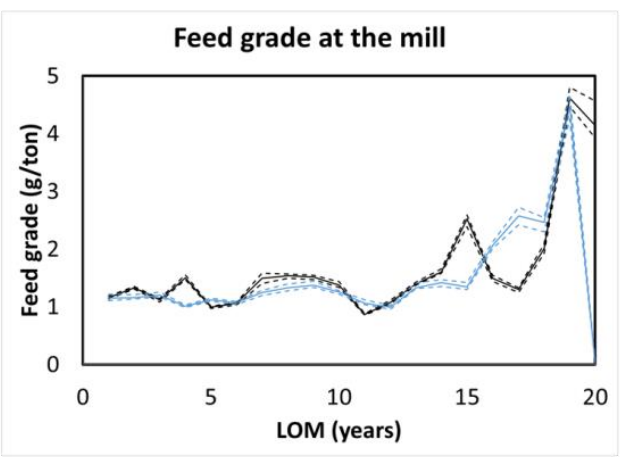

b)

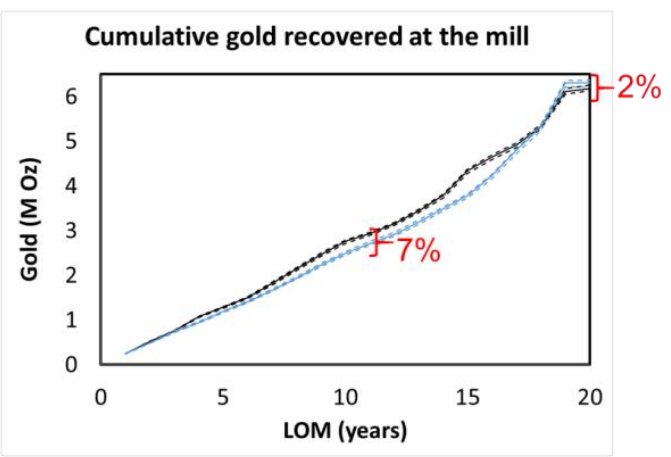

c)

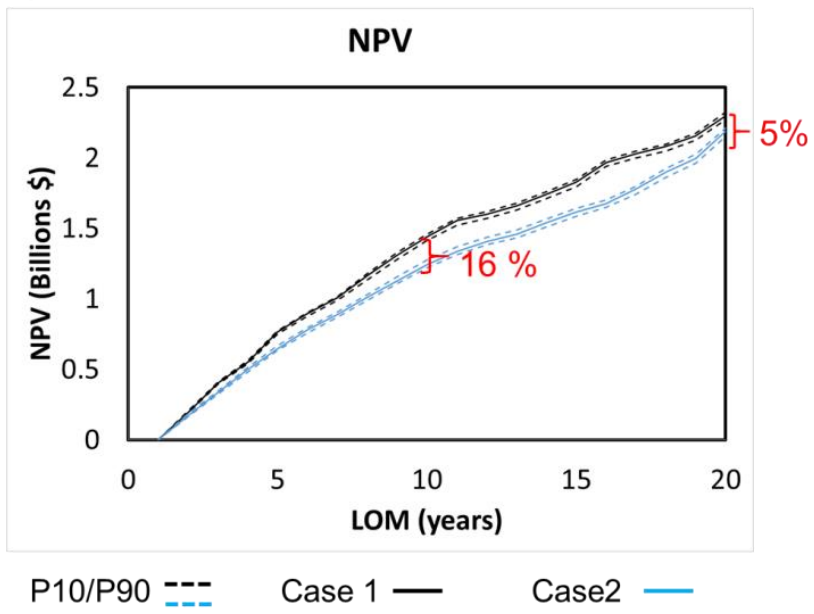

Figure 10. Risk analysis of production forecasts for (a) head grade at the mill; (b) cumulative gold recovered at the mill and (c) NPV assessment. P10 and P90 representing the 10th and 90th percentiles, respectively. 


\section{Conclusions}

This paper investigates the effects of using high-order simulations of an ore deposit in the simultaneous stochastic optimization of a gold mining complex. The high-order simulations are generated directly at the block-support scale and are used as inputs to the simultaneous optimization (Case 1). The optimized LOM production schedule generated is benchmarked against a case where the stochastic realizations of the orebody are generated through the use of a conventional second-order simulation method, SGS (Case 2). The geological realizations from both methods present comparable proportions of tonnages and grades, but very different spatial connectivity of high-grades. Frameworks based on the second-order statistics, in particular, Gaussian-based, maximize the spatial disorder of the generated realizations preventing the reproduction of the connectivity of high-grades.

In this study, the high-order simulations present a greater degree of continuity of high-grades, which is notably more pronounced in the $\mathrm{NE} / 45^{\circ}$ direction when compared to the second-order simulations. This information is incorporated into the simultaneous stochastic optimization framework driving the sequence of extraction favoring this direction. The direct consequence of the above is that Case 1 favors the zones of more connected high-grades, which are processed together and increase the mill's head grade. The result is that a higher amount of metal is produced earlier, with, most notably, $7 \%$ more gold being recovered by the end of year 10 . This is achieved while mining less waste-the strip ratio of Case 1 being consistently below Case 2 . The combined effect of producing more metal earlier and mining less waste increases the NPV by $5 \%$ to $16 \%$ when compared to Case 2 . These findings demonstrate that high-order simulation methods play a significant role in mining complex optimization and that the simultaneous stochastic optimization of a mining complex can profit from the benefits of using simulations that can reproduce the multi-point connectivity of high-grades.

Future work will focus on extending the high-order block support simulation method to incorporate correlated elements into a single framework and will continue to improve the related computation efficiency. Additional case studies in different mining complexes and commodities will further assist related effects and applied aspects.

Author Contributions: Conceptualization, J.P.d.C. and R.D.; Methodology, J.P.d.C. and R.D.; Software, J.P.d.C.; Validation J.P.d.C. and R.D.; Formal analysis, J.P.d.C. and R.D.; Investigation, J.P.d.C.; Resources, R.D.; Data curation, J.P.d.C.; Writing—original draft preparation J.P.d.C. and R.D.; Writing-review and editing, R.D.; Visualization, J.P.d.C.; Supervision, R.D.; Project administration, R.D.; Funding acquisition, R.D.

Funding: This work is funded by the National Science and Engineering Research Council of Canada (NSERC) CRD Grant CRDPJ 500414-16, NSERC Discovery Grant 239019, the COSMO mining industry consortium (AngloGold Ashanti, Barrick Gold, BHP, De Beers, IAMGOLD, Kinross, Newmont Mining and Vale), and the IAMG by the 2017 Mathematical Geosciences Student Award

Acknowledgments: Many thanks are in order to Leonardo Fusciardi of DeBeers, for his valuable comments on an earlier version of this manuscript.

Conflicts of Interest: The authors declare no conflict of interest.

\section{References}

1. Goodfellow, R.; Dimitrakopoulos, R. Simultaneous stochastic optimization of mining complexes and mineral value chains. Math. Geosci. 2017, 49, 341-360. [CrossRef]

2. Montiel, L.; Dimitrakopoulos, R. Optimizing mining complexes with multiple processing and transportation alternatives: An uncertainty-based approach. Eur. J. Oper. Res. 2015, 247, 166-178. [CrossRef]

3. Pimentel, B.S.; Mateus, G.R.; Almeida, F.A. Mathematical models for optimizing the global mining supply chain. In Intelligent Systems in Operations: Methods, Models and Applications in the Supply Chain; Nag, B., Ed.; IGI Global: Hershey, PA, USA, 2010; pp. 133-163. [CrossRef]

4. Montiel, L.; Dimitrakopoulos, R. Stochastic mine production scheduling with multiple processes: Application at Escondida Norte, Chile. J. Min. Sci. 2013, 49, 583-597. [CrossRef]

5. Montiel, L.; Dimitrakopoulos, R. Simultaneous stochastic optimization of production scheduling at Twin Creeks Mining Complex, Nevada. Min. Eng. 2018, 70, 12-20. [CrossRef] 
6. Goodfellow, R.C.; Dimitrakopoulos, R. Global optimization of open pit mining complexes with uncertainty. Appl. Soft Comput. J. 2016, 40, 292-304. [CrossRef]

7. Del Castillo, M.F.; Dimitrakopoulos, R. Dynamically optimizing the strategic plan of mining complexes under supply uncertainty. Resour. Policy 2019, 60, 83-93. [CrossRef]

8. Hoerger, S.; Hoffman, L.; Seymour, F. Mine planning at Newmont's Nevada operations. Min. Eng. 1999, 51, 26-30.

9. Stone, P.; Froyland, G.; Menabde, M.; Law, B.; Pasyar, R.; Monkhouse, P. Blasor-blended iron ore mine planning optimization at Yandi, Western Australia. In Orebody Modelling and Strategic Mine Planning: Uncertainty and Risk Management Models; Dimitrakopoulos, R., Ed.; Spectrum Series, 14; AusIMM: Carlton, Australia, 2007; Volume 14, pp. 133-136.

10. Zuckerberg, M.; Stone, P.; Pasyar, R.; Mader, E. Joint ore extraction and in-pit dumping optimisation. In Orebody Modelling and Strategic Mine Planning; Dimitrakopoulos, R., Ed.; Spectrum Series, 14; AusIMM: Carlton, Australia, 2007; pp. 137-140.

11. Menabde, M.; Froyland, G.; Stone, P.; Yeates, G.A. Mining schedule optimisation for conditionally simulated orebodies. In Orebody Modelling and Strategic Mine Planning; Dimitrakopoulos, R., Ed.; Spectrum Series, 14; AusIMM: Carlton, Australia, 2007; Volume 14, pp. 379-384.

12. Whittle, G. Global asset optimization. In Orebody Modelling and Strategic Mine Planning: Uncertainty and Risk Management Models; Dimitrakopoulos, R., Ed.; Spectrum Series, 14; AusIMM: Carlton, Australia, 2007; pp. 331-336.

13. Whittle, J. The global optimiser works-What next. In Advances in Applied Strategic Mine Planning; Dimitrakopoulos, R., Ed.; Springer International Publishing: Cham, Switzerland, 2018; pp. 31-37. [CrossRef]

14. Topal, E.; Ramazan, S. Strategic mine planning model using network flow model and real case application. Int. J. Min. Reclam. Environ. 2012, 26, 29-37. [CrossRef]

15. Khan, A.; Asad, M.W.A. A mathematical programming model for optimal cut-off grade policy in open pit mining operations with multiple processing streams. Int. J. Min. Reclam. Environ. 2018, 1-10. [CrossRef]

16. Vallée, M. Mineral resource + engineering, economic and legal feasibility = ore reserve. CIM Bull. 2000, 93, 53-61.

17. Dowd, P. Risk assessment in reserve estimation and open-pit planning. Trans. Inst. Min. Metall. 1994, 103, 148-154. [CrossRef]

18. Ravenscroft, P.J. Risk analysis for mine scheduling by conditional simulation. Trans. Inst. Min. Met. Sec. A Min. Ind. 1992, 101. [CrossRef]

19. Dimitrakopoulos, R.; Farrelly, C.T.; Godoy, M. Moving forward from traditional optimization: Grade uncertainty and risk effects in open-pit design. Min. Technol. 2002, 111, 82-88. [CrossRef]

20. Dimitrakopoulos, R. Stochastic optimization for strategic mine planning: A decade of developments. J. Min. Sci. 2011, 47, 138-150. [CrossRef]

21. Journel, A.G.; Huijbregts, C.J. Mining Geostatistics; Blackburn Press: New York, NY, USA, 1978.

22. David, M. Handbook of Applied Advanced Geostatistical Ore Reserve Estimation; Developments in geomathematics; Elsevier: Amsterdam, The Netherlands, 1988.

23. Goovaerts, P. Geostatistics for Natural Resources Evaluation; Applied geostatistics series; Oxford University Press: New York, NY, USA, 1997.

24. Mariethoz, G.; Caers, J. Multiple-Point Geostatistics: Stochastic Modeling with Training Images; Wiley: Hoboken, NJ, USA, 2014. [CrossRef]

25. Rossi, M.E.; Deutsch, C.V. Mineral Resource Estimation; Springer: Dordrecht, The Netherlands, 2014. [CrossRef]

26. Wang, H.; Wang, X.; Wellmann, J.F.; Liang, R.Y. Bayesian stochastic soil modeling framework using Gaussian Markov random fields. ASCE-ASME J. Risk Uncertain. Eng. Syst. Part A Civ. Eng. 2018, 4, 04018014. [CrossRef]

27. Journel, A.G. Modeling uncertainty: Some conceptual thoughts. In Geostatistics for the Next Century; Dimitrakopoulos, R., Ed.; Springer: Dordrecht, The Netherlands, 1994; pp. 30-43. [CrossRef]

28. Nussbaumer, R.; Mariethoz, G.; Gloaguen, E.; Holliger, K. Which path to choose in sequential gaussian simulation. Math. Geosci. 2018, 50, 97-120. [CrossRef]

29. Godoy, M. The Effective Management of Geological Risk in Long-Term Production Scheduling of Open Pit Mines. Ph.D. Thesis, University of Queensland, Brisbane, QLD, Australia, 2003. 
30. Boucher, A.; Dimitrakopoulos, R. Block simulation of multiple correlated variables. Math. Geosci. 2009, 41, 215-237. [CrossRef]

31. Journel, A.G. Roadblocks to the evaluation of ore reserves-the simulation overpass and putting more geology into numerical models of deposits. In Advances in Applied Strategic Mine Planning; Dimitrakopoulos, R., Ed.; Springer: Cham, The Netherlands, 2018; pp. 47-55. [CrossRef]

32. Journel, A.G.; Deutsch, C.V. Entropy and spatial disorder. Math. Geol. 1993, 25, 329-355. [CrossRef]

33. Guardiano, F.B.; Srivastava, R.M. Multivariate geostatistics: Beyond bivariate moments. In Geostatistics Tróia '92: Volume 1; Soares, A., Ed.; Springer: Dordrecht, The Netherlands, 1993; pp. 133-144. [CrossRef]

34. Strebelle, S. Conditional simulation of complex geological structures using multiple-point statistics. Math. Geol. 2002, 34, 1-21. [CrossRef]

35. Journel, A.G. Beyond Covariance: The Advent of Multiple-Point Geostatistics; Leuangthong, O., Deutsch, C.V., Eds.; Springer: Dordrecht, The Netherlands, 2005; Volume 14. [CrossRef]

36. Zhang, T.; Switzer, P.; Journel, A. Filter-based classification of training image patterns for spatial simulation. Math. Geol. 2006, 38, 63-80. [CrossRef]

37. Arpat, G.B.; Caers, J. Conditional simulation with patterns. Math. Geol. 2007, 39, 177-203. [CrossRef]

38. Mariethoz, G.; Renard, P.; Straubhaar, J. The direct sampling method to perform multiple-point geostatistical simulations. Water Resour. Res. 2010, 46, 1-14. [CrossRef]

39. Chatterjee, S.; Mustapha, H.; Dimitrakopoulos, R. Fast wavelet-based stochastic simulation using training images. Comput. Geosci. 2016, 20, 399-420. [CrossRef]

40. Straubhaar, J.; Renard, P.; Mariethoz, G.; Chugunova, T.; Biver, P. Fast and interactive editing tools for spatial models. Math. Geosci. 2019, 51, 109-125. [CrossRef]

41. Santibañez, F.; Silva, J.F.; Ortiz, J.M. Sampling strategies for uncertainty reduction in categorical random fields: Formulation, mathematical analysis and application to multiple-point simulations. Math. Geosci. 2019, 1-46. [CrossRef]

42. Shahraeeni, M. Enhanced multiple-point statistical simulation with backtracking, forward checking and conflict-directed backjumping. Math. Geosci. 2019, 51, 155-186. [CrossRef]

43. Goodfellow, R.; Albor Consuegra, F.; Dimitrakopoulos, R.; Lloyd, T. Quantifying multi-element and volumetric uncertainty, Coleman McCreedy deposit, Ontario, Canada. Comput. Geosci. 2012, 42, 71-78. [CrossRef]

44. Osterholt, V.; Dimitrakopoulos, R. Simulation of orebody geology with multiple-point geostatistics-application at Yandi channel iron ore deposit, WA, and implications for resource uncertainty. In Orebody Modelling and Strategic Mine Planning; Dimitrakopoulos, R., Ed.; Spectrum Series, 14; AusIMM: Carlton, Australia, 2007; Volume 14, pp. 51-60.

45. Dimitrakopoulos, R.; Mustapha, H.; Gloaguen, E. High-order statistics of spatial random fields: Exploring spatial cumulants for modeling complex non-Gaussian and non-linear phenomena. Math. Geosci. 2010, 42, 65-99. [CrossRef]

46. Mustapha, H.; Dimitrakopoulos, R. HOSIM: A high-order stochastic simulation algorithm for generating three-dimensional complex geological patterns. Comput. Geosci. 2011, 37, 1242-1253. [CrossRef]

47. Minniakhmetov, I.; Dimitrakopoulos, R. Joint high-order simulation of spatially correlated variables using high-order spatial statistics. Math. Geosci. 2017, 49, 39-66. [CrossRef]

48. de Carvalho, J.P.; Dimitrakopoulos, R.; Minniakhmetov, I. High-order block support spatial simulation method and its application at a gold deposit. Math. Geosci. 2019, 1-18. [CrossRef]

49. Minniakhmetov, I.; Dimitrakopoulos, R.; Godoy, M. High-order spatial simulation using Legendre-like orthogonal splines. Math. Geosci. 2018, 50, 753-780. [CrossRef]

50. Yao, L.; Dimitrakopoulos, R.; Gamache, M. A new computational model of high-order stochastic simulation based on spatial Legendre moments. Math. Geosci. 2018, 50, 929-960. [CrossRef]

51. Gómez-Hernández, J.J.; Wen, X.H. To be or not to be multi-Gaussian? A reflection on stochastic hydrogeology. Adv. Water Resour. 1998, 21, 47-61. [CrossRef]

52. Journel, A.G.; Alabert, F. Non-gaussian data expansion in the earth sciences. Terra Nova 1989, 1, $123-134$. [CrossRef]

53. Renard, P.; Allard, D. Connectivity metrics for subsurface flow and transport. Adv. Water Resour. 2013, 51, 168-196. [CrossRef] 
54. Qureshi, S.E.; Dimitrakopoulos, R. Comparison of stochastic simulation algorithms in mapping spaces of uncertainty of non-linear transfer functions. In Geostatistics Banff 2004, Quantitative Geology and Geostatistics, Vol. 14; Leuangthong, O., Deutsch, C.V., Eds.; Springer: Dordrecht, The Netherlands, 2005; pp. 959-968.

55. Dimitrakopoulos, R.; Godoy, M. Grade control based on economic ore/waste classification functions and stochastic simulations: Examples, comparisons and applications. Min. Technol. 2014, 123, 90-106. [CrossRef]

56. Montiel, L.; Dimitrakopoulos, R. A heuristic approach for the stochastic optimization of mine production schedules. J. Heuristics 2017, 23, 397-415. [CrossRef]

57. Kumar, A.; Dimitrakopoulos, R. Application of simultaneous stochastic optimization with geometallurgical decisions at a copper-gold mining complex. Min. Technol. 2019, 1-18. [CrossRef]

58. Dimitrakopoulos, R.; Luo, X. Generalized sequential Gaussian simulation on group size v and screen-effect approximations for large field simulations. Math. Geol. 2004, 36, 567-590. [CrossRef]

59. Johnson, M.E. Multivariate Statistical Simulation; Wiley Series in Probability and Statistics; John Wiley \& Sons, Inc.: Hoboken, NJ, USA, 1987. [CrossRef]

60. Wei, Y.; Wang, G.; Yang, P. Legendre-like orthogonal basis for spline space. CAD Comput. Aided Des. 2013, 45, 85-92. [CrossRef]

61. Isaaks, E.H. The Application of Monte Carlo Methods to the Analysis of Spatially Correlated Data. Ph.D. Thesis, Stanford University, Stanford, CA, USA, 1990.

62. Dimitrakopoulos, R.; Ramazan, S. Uncertainty-based production scheduling in open pit mining. SME Trans. 2004, 316, 106-112.

63. Saliba, Z.; Dimitrakopoulos, R. Simultaneous stochastic optimization of an open pit gold mine complex with supply and market uncertainty. In COSMO Research Report No 11, Vol. 1; Dimitrakopoulos, R., Ed.; COSMO-Stochastic Mine Planning Laboratory: Montreal, QC, Canada, 2017; pp. 173-200.

64. Lamghari, A.; Dimitrakopoulos, R. A diversified Tabu search approach for the open-pit mine production scheduling problem with metal uncertainty. Eur. J. Oper. Res. 2012, 222, 642-652. [CrossRef]

65. Lamghari, A.; Dimitrakopoulos, R.; Ferland, J.A. A variable neighbourhood descent algorithm for the open-pit mine production scheduling problem with metal uncertainty. J. Oper. Res. Soc. 2014, 65, 1305-1314. [CrossRef]

66. Lamghari, A.; Dimitrakopoulos, R. Hyper-heuristic approaches for strategic mine planning under uncertainty. Comput. Oper. Res. 2018, in press. [CrossRef]

(C) 2019 by the authors. Licensee MDPI, Basel, Switzerland. This article is an open access article distributed under the terms and conditions of the Creative Commons Attribution (CC BY) license (http://creativecommons.org/licenses/by/4.0/). 